\title{
VENTAJAS Y DESVENTAJAS DE USAR LABORATORIOS VIRTUALES EN EDUCACIÓN A DISTANCIA: LA OPINIÓN DEL ESTUDIANTADO EN UN PROYECTO DE SEIS AÑOS DE DURACIÓN
}

\author{
Julián Monge Nájera \\ Director de la Revista de Biología Tropical, Universidad de Costa Rica \\ San José, Costa Rica \\ Victor Hugo Méndez Estrada \\ Investigador del Centro para el Mejoramiento de los Procesos Académicos (CEMPA), \\ Universidad Estatal a Distancia \\ San José, Costa Rica
}

Recibido 21-V-2007 • Aceptado 12-VI-2007

\begin{abstract}
Resumen: Con el fin de evaluar los laboratorios virtuales utilizados por el estudiantado de ciencias de la educación a distancia en la UNED de Costa Rica, durante seis años se encuestó el nivel de satisfacción en cada curso a los usuarios de 12 laboratorios virtuales. Los resultados se analizan en dos contextos: la opinión del personal docente que usó los laboratorios y los parámetros internacionales de lo que debe ser un laboratorio virtual. La tasa de aprobación de los contenidos, los aspectos informáticos, el diseño gráfico, la interactividad y la eficacia de los laboratorios virtuales superó el $75 \%$ en todos los laboratorios. Al no haber diferencia estadísticamente significativa en la tasa de estudiantes que aprobaron el curso tras ejecutar laboratorios reales o virtuales, consideramos que los laboratorios virtuales son una buena opción para quienes no pueden o no desean realizar prácticas en un laboratorio real. Representan además una opción creativa, moderna y económica para instituciones universitarias, tanto a distancia como presenciales, que requieran laboratorios dentro de sus procesos de formación.
\end{abstract}

Palabras clave: Laboratorios virtuales, educación a distancia, opinión de estudiantes, ventajas, desventajas, requisitos.
Introducción: el papel de las TIC en la enseñanza mediante laboratorios

La enseñanza de la parte práctica de las asignaturas del área de las ciencias naturales preocupa a los docentes en todo el mundo, ya que desde el renacimiento se ha considerado necesario complementar la enseñanza teórica del aula con la ejecución de experimentos. Las prácticas de laboratorio permiten a la persona que estudia manipular materiales, instrumentos e ideas, así como aplicar su propia iniciativa y originalidad. Pero en los sistemas de enseñanza a distancia es difícil la adecuada incorporación de los cursos prácticos a la actividad cotidiana de los estudiantes, al tener que presentarse en un sitio y horario definidos, a pesar de que el resto del aprendizaje se realiza con mayor libertad de horario y lugar. Es aquí donde entran en juego los laboratorios simulados mediante las tecnologías de la información y comunicación (TIC). Sin embargo, también en la educación presencial los laboratorios 


\begin{abstract}
In order to evaluate the virtual laboratories used by Science Teaching students at UNED, the Costa Rican distance education university, we surveyed students who used a total of 12 virtual laboratories for six years. We wanted to analyze a longer time-span than most studies have accomplished, which usually refer to briefer periods. Results are analyzed in two contexts: the opinion of a second source (teachers and tutors who used the laboratories) and international standards for a virtual laboratory. The approval rate for contents, the informatics aspects, the graphic design, the interactivity, and the efficacy of the virtual laboratories was rated positively by $75 \%$ or more of the students. There was no difference in student success in course groups that used real laboratories and those that used virtual laboratories, so we conclude that virtual laboratories are a good option for those who cannot (or do not want to) use real laboratory experiments. Furthermore, virtual laboratories are a creative, modern, and economical option for both traditional and distance universities and institutes of technology.
\end{abstract}

Key words: Virtual laboratories, distance education, student opinion, advantages, requirements. representan un reto, como explicaremos más adelante.

Las universidades a distancia están probando las nuevas opciones que ofrece el mercado, como el uso de las TIC. Pero tanto en los países industrializados como en los países en proceso de industrialización, existen diversos informes que indican que el uso adecuado de las TIC aún es insatisfactorio desde el punto de vista del proceso enseñanza-aprendizaje (Monge Nájera y otros, 2001). Un ejemplo de esto es la Universidad Estatal a Distancia de Costa Rica (UNED), en la cual varios estudios han concluido que se debe avanzar más en el uso de las actuales tecnologías, ya que los esfuerzos realizados son insuficientes y falta aún mucho camino por recorrer (Monge Nájera y otros, 2001). Además, existen pocos casos concretos donde se ha evaluado el uso de las computadoras en la enseñanza a distancia, siendo los laboratorios virtuales uno de los mejor estudiados (Montero y otros, 1999; Rivas, 1999a; Cruz, 1998; Rodino, 1996, 1997; Monge Nájera y otros, 2002).

En relación con las TIC en general y los laboratorios virtuales en particular, se ha concluido que se debe hacer un esfuerzo adicional para eliminar la brecha entre mujeres y hombres, ya que estas sufren una discriminación fuerte en cuanto a acceso a computadores y muy fuerte en cuanto al uso de Internet (Monge Nájera y otros, 1999). El intento de corregir esa situación debe ser prioritario en aquellos centros universitarios que no cuentan con tutoría presencial o laboratorios reales (Monge Nájera y otros, 1999).

Repetidamente se ha expresado en la UNED el deseo de que la institución se mantenga actualizada en cuanto a tendencias tecnológicas que favorezcan sus objetivos educacionales y le permitan competir con las demás universidades públicas del país, que están incorporando paulatinamente a la docencia TIC semejantes a las que la UNED operó de manera pionera hace una década. Este aspecto ha sido 
analizado en varias oportunidades por funcionarios de distintas dependencias de la UNED (Méndez Estrada, 1999a, b; Monge Nájera, 1999, Rivas, 1999a, b), quienes han concluido que tanto la UNED como las universidades presenciales costarricenses deben ampliar la variedad de sus materiales de aprendizaje, incluyendo más cursos que utilicen nuevas tecnologías, lo cual es necesario porque sus egresados(as) hallarán estas tecnologías en su vida laboral. Se advierte, sin embargo, que esta incorporación de TIC debe hacerse con el mismo rigor científico, pedagógico y técnico que se siguió en la producción de los laboratorios virtuales. Estos laboratorios fueron desarrollados inicialmente de forma independiente por la UNED y ampliados posteriormente con el apoyo financiero del Centro de Aplicación de Estudios y Recursos al Aprendizaje a Distancia (CAERENAD) canadiense, para uso no solo en Costa Rica sino en África, Canadá y Sudamérica (Rivas, 1999b).

\section{Laboratorios virtuales: qué son y cómo difiere su uso en países industrializados y países en vías de industrialización}

En computación, el término virtual significa "que no es real". En general distingue algo que es meramente conceptual de algo que es físicamente real, y se puede usar en una amplia variedad de situaciones. Los laboratorios virtuales han sido definidos como una simulación en computadora de una amplia variedad de situaciones, desde prácticas manipulables hasta visitas guiadas, en un ambiente interactivo que quienes aprenden pueden usar fuera del campus universitario y sin ayuda de personal docente.

Típicamente el lugar del laboratorio virtual es la computadora personal, donde no se mantiene una comunicación directa con personal docente, pues el laboratorio virtual tradicional ha sido estructurado de tal forma que pueda hacerse desde cualquier computadora personal, sin necesidad de conexión a Internet, aunque también hay casos de prácticas de laboratorio dentro de lecciones virtuales de universidades a distancia en que sí hay comunicación directa con personal docente e incluso con otras personas que realizan la práctica simultáneamente (Bailey y Lluetkehans, 1998; Cisneros, 1998; Laaser, 1998; Pensa y Sabulsky, 1998; Tapani, 1998).

Dado que un laboratorio virtual es una práctica que se observa en la pantalla del computador, solo existe como información binaria. Normalmente cuenta también con un manual de laboratorio, pero este suele ser igualmente electrónico. La comunicación entre el personal docente y quien aprende suele ser a través del manual electrónico, quedando la opción para quien tiene dudas de usar el teléfono, correo electrónico o un medio similar (Sowizral y otros, 2000; Sebastián y otros, 2003).

Curiosamente, los laboratorios virtuales, que originalmente fueron desarrollados dentro de programas espaciales y militares dotados de enormes presupuestos (Monge Nájera, 1998, Dormido y otros, 2000, Gil y otros, 2003), se convirtieron con los años en la opción para quienes sufren de lo contrario: presupuestos pequeños. Un caso extremo es el de Cuba, donde según Alejandro (2004) el estudiantado debe trabajar en laboratorios reales que son obsoletos y carecen de mantenimiento y acceso a Internet.

En Europa también hay experiencias de laboratorios virtuales, pero dentro de un contexto muy diferente, pues frecuentemente no se trata de enfrentar problemas de laboratorios inadecuados o dificultades de acceso, sino de proteger al estudiantado de peligros asociados a productos químicos o aparatos mecánicos, a la vez que se protege a los equipos de daños por impericia durante el proceso de aprendizaje (Salzmann y otros, 1999; Candelas y otros, 2003, 2004a, b).

Otra variación de este tema es la UNED, donde los laboratorios reales son relativamente actualizados y aceptablemente mantenidos, pero un porcentaje del 
estudiantado tiene conflictos de horario o geográficos que les dificultan usarlos. Por ello, la UNED usa laboratorios virtuales como una opción que libera al estudiantado de horarios fijos y desplazamientos costosos o prolongados. Además, la Universidad ahorra una gran cantidad de recursos pues es mucho más barato producir un laboratorio virtual (distribuible mediante red, disco o similar) que mantener la infraestructura, equipo y personal de un laboratorio real (Monge Nájera y otros, 1998, 2001, 2002, según se detallará más adelante).

\section{Laboratorios reales y su diferencia con los laboratorios virtuales en la UNED}

Muchas de las materias contempladas dentro del plan curricular en la UNED cuentan con una parte teórica y otra práctica. La modalidad práctica normalmente consiste en la realización de un laboratorio. Cada práctica de laboratorio se lleva a cabo en una fecha prefijada, con horario fijo y en la planta física de uno de los centros universitarios, previamente establecidos por la coordinación del Programa de Laboratorios de la UNED (Monge Nájera y otros, 2002).

Estas actividades de laboratorio cuentan con un valor porcentual significativo dentro de la evaluación del curso. Una ventaja de las prácticas en el laboratorio real es que se mantiene un contacto "cara a cara”, tanto del estudiantado entre sí como del personal docente y administrativo con el estudiantado. Sin embargo, se ha criticado que en algunos casos ese contacto se vuelve algo impersonal debido a que en una misma sesión de laboratorio se realizan hasta tres prácticas, porque con la modalidad de cuatrimestres se reduce el tiempo para las tutorías presenciales (Monge Nájera y otros, 1998).

El material didáctico de las materias con laboratorio incluye un manual impreso que describe todas las prácticas a desarrollar en el curso. Los laboratorios son estructurados de manera que se estudie con anticipación cada una de ellas, para saber qué hacer al llegar al laboratorio. Como es típico de las actividades de laboratorio en todas partes, hay que producir un informe escrito, según el formato establecido por cada cátedra; normalmente, éste incluye resultados, discusión, conclusiones y respuestas a un cuestionario (Gillet y otros, 2001, Monge Nájera y otros, 2002, Puente y otros, 2002).

La realización del laboratorio consiste, por ejemplo, en aplicar una sustancia a un tejido, para luego observar lo que sucede, ya sea al microscopio o a simple vista. Sin embargo, en la UNED suele dejarse la manipulación y preparación de reactivos, así como la preparación de láminas para microscopio, a un ayudante de laboratorio contratado.

La técnica de laboratorios virtuales, por otra parte, no requiere asistir a un espacio físico, o sea, al "laboratorio" del centro universitario, para realizar las prácticas, ni existe para su ejecución un horario establecido (Gillet y otros, 1997). En la UNED, las estudiantes y los estudiantes reciben un disco compacto con todos los laboratorios del curso, y unas hojas impresas que explican cómo usarlo. Sin embargo, están diseñados de manera que basta insertar el disco en la computadora: el laboratorio se inicia automáticamente y funciona en cualquier computadora personal dotada de un programa para navegar en Internet. El uso de los laboratorios virtuales permite generar nuevos espacios pedagógicos interactivos, donde se promueve la participación interactiva con los contenidos de cada laboratorio; facilitándose la construcción del conocimiento, así como el almacenamiento, transmisión, recuperación, aplicación y enriquecimiento de los contenidos. Todo ello ocurre en forma autosuficiente, pues se brinda la posibilidad de un aprendizaje individual, al propio ritmo y adaptado a las necesidades de la vida cotidiana, obviando costos de traslado, alimentación y hospedaje. No solamente pueden ejecutarse en el momento más conveniente, sin la presión de compañeros y 
docentes, o sufriendo límites de disponibilidad de microscopios, equipos de medición y otros, sino que se practica la habilidad en el uso de simulaciones computarizadas. Ello se facilita por el hecho de que la manipulación del laboratorio virtual es muy sencilla. El diseño se hizo con el lenguaje de computación llamado HTML, lo cual permite navegar fácilmente, su interfaz es amigable y su diseño cuidadoso permite usarlo en computadoras con poco espacio en disco y de cualquier sistema (Windows, Macintosh, Linux, etc.)

$\mathrm{Su}$ potencial es grande para combinar esquemas o fotografías de órganos, sistemas, etc., observarse vídeos de experimentos, simular situaciones de movimiento molecular, desarrollo embrionario, cambios ecológicos, etc. Los laboratorios virtuales pueden aplicarse en los siguientes campos (Bailey y Lluetkehans, 1998):

- Laboratorios de ciencias, tanto biológicas como químicas, físicas $\mathrm{y}$ agronómicas.

- $\quad$ En cursos donde se requiera enseñar procesos o eventos que tardan mucho tiempo en ocurrir.

- $\quad$ Cuando se trate de situaciones peligrosas a las que no querríamos exponer al estudiantado (exploración de cavernas, volcanes y profundidades acuáticas; manipulación de sustancias químicas venenosas o explosivas, etc.).

- En cursos que requieran de giras, las cuales se podrán hacer virtuales, evitando los costos de viaje, alimentación y alojamiento, así como el peligro de accidentes en el campo o laborales.

- $\quad$ En casos en donde se quiera demostrar el comportamiento animal, el crecimiento de plantas o cultivos, los estragos de los cambios ambientales, que no ocurren normalmente o que son dañinos para ecosistemas y organismos.

- En cursos de capacitación en el uso de equipos (fue en este campo que surgieron los laboratorios virtuales, usados en adiestramiento de cosmonautas y pilotos).

En breve, el uso de laboratorios virtuales permite obtener los siguientes beneficios (Méndez y otros, 2001):

- Ampliar la cobertura de los cursos.

- Disminuir los costos de traslado, alimentación y hospedaje de los estudiantes.

- Simular situaciones que en realidad tendría escasas posibilidades de realizarlas.

- Repetir los eventos o fenómenos cuantas veces se requiera.

- Relacionar fenómenos con sus consecuencias.

- Desarrollar habilidades en el uso de la computadora.

\section{Origen e implementación de los laboratorios virtuales en la UNED}

En la UNED, el programa de laboratorios virtuales nació en 1997 como una iniciativa de Víctor Hugo Méndez, Julián Monge y Marta Rivas, quienes eran científicos del Centro de Investigación Académica (CIAC), posteriormente convertido en el Centro de Mejoramiento para los Procesos Académicos (CEMPA). En los primeros años se elaboraron cuatro laboratorios: 1) Digestión, 2) Nutrición, 3) Reproducción y 4) Tejidos. Estos laboratorios fueron planeados como alternativa de enseñanza a distancia para el estudiantado de los cursos de ciencias biológicas y para incorporar las tecnologías de la información y comunicación (TIC) a la unidad didáctica modular, medio usado para la difusión de los conocimientos en la UNED (Suárez, 1998).

Para el tercer cuatrimestre de 1999, a dos años de iniciado el proyecto, ya se estaban evaluando tanto el formato como el contenido de estos laboratorios virtuales con dos objetivos, conocer la opinión estudiantil sobre el formato y contenido de 
los laboratorios, y aprovechar esa información para elaborar versiones mejoradas de los laboratorios. Efectivamente, las opiniones de quienes usaron esos primeros laboratorios virtuales sirvieron de base para mejorarlos. También fueron consideradas en la elaboración de los laboratorios posteriores, auspiciados por el programa CAERENAD (cooperación canadiense) y por la UNED.

En 2004 se concluyó la primera fase de producción, con 12 laboratorios virtuales, los cuatro mencionados anteriormente y los siguientes, que se produjeron en versiones equivalentes en tres idiomas: español, inglés y francés. A lo largo de la experiencia con los laboratorios virtuales se han elevado paulatinamente sus niveles de complejidad, incorporado en su programación los nuevos aportes tecnológicos que iban apareciendo.

Los laboratorios quinto a onceavo fueron: 5) Ecología: la trama de la vida, 6) El mundo de las mariposas, 7) El mundo de las orquídeas, 8) La evolución y las aves, 9) Modelos ecológicos de depredador y presa, y 11) Biodiversidad. El último, 12) La célula (en español y francés), se elaboraron en conjunto con la Universidad de Québec.

Para conocer la composición detallada de los laboratorios y las opiniones del personal docente, recomendamos consultar los artículos previamente publicados al respecto (e.g. Monge Nájera, 1998, Monge Nájera y otros, 1999, 2001). En este trabajo presentamos información nueva, fundamentalmente las opiniones del estudiantado y nuestras propias lecciones aprendidas a lo largo de seis años durante la elaboración, prueba y mejoramiento de los 12 laboratorios, que en total representan cerca de un centenar de prácticas individuales, cada una enseñando un concepto o una habilidad en el campo de las ciencias naturales.

\section{Justificación del estudio}

La Cátedra de Ciencias Biológicas tiene a su cargo varios cursos, los cuales incluyen en su programación el desarrollo de varias prácticas de laboratorio, entre ellos: Biología General, Botánica General, Zoología General y Biología I, II y III. En los tres primeros cursos, debe matricularse simultáneamente los laboratorios respectivos y asistir quincenalmente al centro universitario a desarrollar las prácticas presenciales de laboratorio.

Las materias Biología I, II y III, no tienen curso de laboratorio paralelo, sin embargo, dentro de su programación está el desarrollo de cuatro prácticas de laboratorio, por lo que hay que trasladarse cuatro veces al centro universitario a realizarlas. El desarrollo de todas estas prácticas de laboratorio ha generado los siguientes problemas, según el estudiantado:

- $\quad$ El traslado obligatorio a los centros universitarios cada quince días, haciendo que estas materias se conviertan en presenciales, lo cual no calza con el objetivo de la educación a distancia, que es precisamente evitar los obstáculos propios de la presencialidad.

- A quienes laboran en el Servicio de Parques Nacionales o como guías turísticos, se les dificulta el traslado al centro universitario, ya que son personas que a menudo se encuentran internadas en algún parque lejano o tienen gira con turistas los sábados y domingos, justo los días que la UNED ofrece los laboratorios en la mayoría de los centros universitarios.

Los laboratorios están regionalizados en San José, Alajuela, Pérez Zeledón, San Carlos, Cañas, Palmares, Cartago, Turrialba y Puntarenas, por lo que las estudiantes y los estudiantes del resto del país deben trasladarse a los centros universitarios más cercanos a realizar sus prácticas de laboratorio. Ello genera cansancio, pérdida de tiempo y gasto de dinero, sobre todo en Guanacaste, Pacífico Sur y Limón. En Guanacaste hay que trasladarse desde Santa Cruz, Liberia, Jicaral y Upala al centro universitario de Cañas, lo que significa en algunos casos 
viajar 4 o 6 horas al día. En la zona sur el problema se agrava pues los centros universitarios de San Vito de Coto Brus y Ciudad Neilly están a más de cuatro horas de distancia de Pérez Zeledón, y eso cuando las condiciones del tiempo son favorables y la carretera es transitable. En Turrialba, el horario de autobuses obliga a pernoctar en la ciudad, con los consiguientes gastos de hotel, alimentación, etc.

Por todo lo anterior, fue necesario establecer el sistema de laboratorios virtuales y evaluarlos mediante este estudio y otros previamente publicados (lista en las referencias).

\section{Planteamiento del problema}

$\mathrm{Al}$ ser los laboratorios virtuales un medio creciente de entregar la docencia en la UNED, es necesario conocer cómo los perciben quienes los usan. En este caso, se trata de la opinión del estudiantado, en una muestra amplia que representa seis años de experiencias, doce laboratorios y cerca de un centenar de prácticas individuales. objetivos:

La presente investigación tiene dos

1) Describir los niveles de complejidad tecnológica en que se encuentran los laboratorios virtuales utilizados por el estudiantado de diversas carreras relacionadas con las ciencias naturales en la UNED.

2) Analizar las críticas, ventajas y desventajas que menciona ese estudiantado sobre los laboratorios virtuales.

\section{Material}

Durante los seis años analizados en este estudio, se distribuyó al estudiantado un cuestionario impreso en el cual se les pedía dar una lista de las ventajas y desventajas que encontraban en cada uno de los laboratorios. Cada grupo respondió tomando en cuenta los siguientes aspectos:
I) Experiencia en computación; II) Facilidad de uso, disfrute del programa, nivel de dificultad y tiempo y III) Aprendizaje de los contenidos de los laboratorios virtuales.

\section{Método}

En esta investigación descriptiva se consideró a todo el estudiantado de la Universidad Estatal a Distancia que ha cursado, desde el 2000, algunas de las materias (Biología General, Botánica, Biología I, Biología II, Biología III, Historia Natural de Costa Rica, Biodiversidad e Inventario de la Naturaleza y Zoología General) con laboratorio virtual. Los laboratorios virtuales se introdujeron en el sistema para facilitar el aprendizaje de quienes no podían o no deseaban asistir a los laboratorios presenciales.

Se distribuyó un cuestionario abierto preguntando (1) ventajas y desventajas percibidas al usar cada laboratorio y (2) si la persona se encontraba o no satisfecha con los laboratorios virtuales en general. Las tutoras y los tutores se encargaban de entregarlo a cada estudiante y luego de recibir las respuestas, las enviaban a los investigadores mediante el sistema de distribución de materiales con que cuenta la UNED. El cuestionario lo respondieron en total 248 estudiantes, refiriéndose a las pruebas con un total de 12 laboratorios a lo largo de seis años.

\section{Resultados}

\section{Opinión del estudiantado sobre los laboratorios virtuales en general}

Más del 75\% de las personas consultadas opinaron como se resume a continuación:

- Las instrucciones dadas para navegar cada laboratorio son claras y no hay problemas importantes con los discos o con la compatibilidad de la computadora usada. 
- $\quad$ La facilidad de uso de los laboratorios virtuales es "buena o excelente", es agradable ejecutarlos y las ilustraciones y demás aspectos gráficos son buenos.

- Los laboratorios ayudan a comprender los contenidos teóricos, pues permiten observar de cerca algunos procesos que son difíciles de estudiar en la naturaleza, como la digestión, la evolución de las aves, o la formación de la membrana celular y sus fenómenos.

- Las preguntas de evaluación ayudan a reforzar el curso teórico pues están planteadas de acuerdo con los objetivos. También permiten profundizar pues motivan a buscar bibliografía complementaria.

\section{Desventajas}

Se mencionan tres problemas básicos, aunque no dominantes, al usar los laboratorios virtuales:

- Dificultad para responder las preguntas de evaluación, dado que varios de los primeros laboratorios no eran autocontenidos y requerían buscar literatura que no estaba disponible en algunas bibliotecas locales.

- $\quad$ El poco conocimiento que tiene una minoría del estudiantado en el uso de programas de computación les hizo algo difícil el comienzo.

- Se requiere mucho tiempo para estudiar y desarrollar cada laboratorio virtual, mientras que los laboratorios reales están restringidos a un tiempo de 2 horas.

\section{Evaluación de laboratorios virtuales específicos}

Aquí se presentan las evaluaciones detalladas y muestras para seis laboratorios, seleccionados de entre el total de 12 por su representatividad. Para cada laboratorio evaluado, presentamos el "formato" (breve descripción del contenido, con algunas imágenes que aparecen en la pantalla) y algunos comentarios a favor y en contra (minoritarios) del laboratorio. Estos comentarios pueden ser contradictorios, pues se eligieron para ser representativos, no por la cantidad de personas que opinaban así: la mayoría de las personas consultadas expresó satisfacción con los laboratorios.

\subsection{La reproducción}

\section{a. Formato}

Se analizan los diversos tipos de reproducción asexual (gemación, regeneración, fisión, partenogénesis y esporulación) y la sexual. Se presentan dibujos y vídeos donde se analiza y ejemplifica cada tipo de reproducción. Estas imágenes corresponden a algunas de las animaciones usadas (Fig. 1).

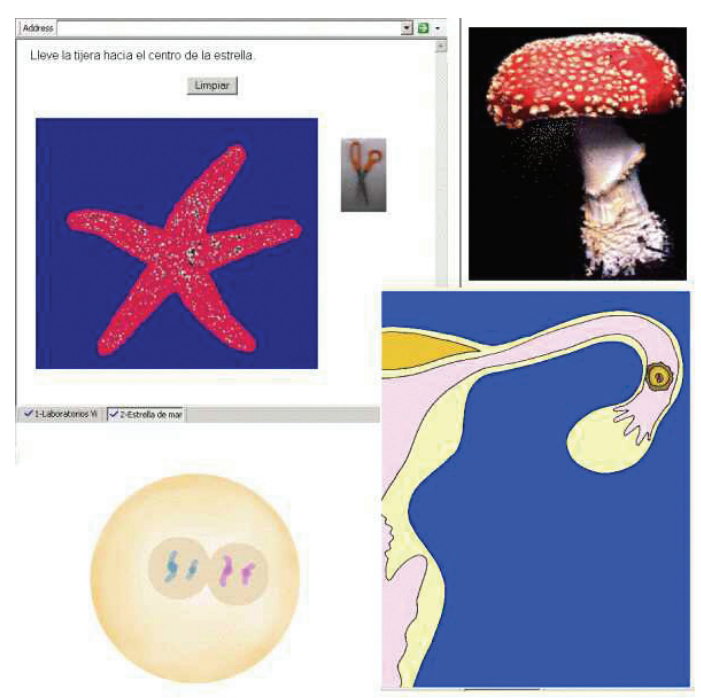

Fig. 1. Cuadros de las animaciones usadas en el laboratorio virtual sobre reproducción orgánica (fisión, esporulación, formación del cigoto y fecundación). 


\section{b. Comentarios a favor}

- $\quad$ Está estructurado con animaciones sencillas, creativas y dinámicas que permiten comprender los diversos aspectos relacionados con la reproducción sexual y asexual.

- $\quad$ El contenido tratado no es extenso, es activo y hay animaciones claras.

- Ofrece buena información concreta y práctica sobre el tema del laboratorio.

b. Comentarios en contra (minoritarios)

- Las actividades son difíciles de realizar.

- Los dibujos no expresan claramente los procesos que se querían mostrar.

\subsection{Tejidos}

\section{a. Formato}

El tema de los tejidos orgánicos se presenta mediante un cuerpo humano, una imagen de un microscopio y fotografías de tejidos vistos en dos niveles de amplificación. Con el ratón de la computadora se puede "desarmar" el cuerpo humano, arrastrando cada tejido hasta el microscopio para observarlo amplificado (Fig. 2).

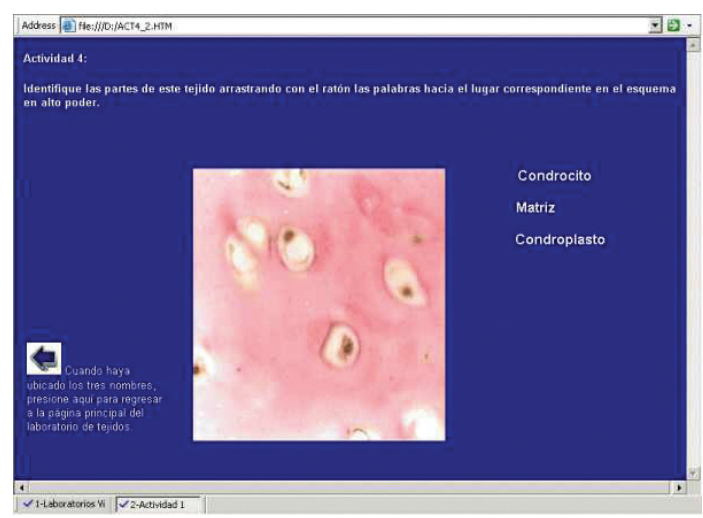

Fig. 2. Pantalla del laboratorio sobre tejidos del cuerpo humano.
Una vez elegido el tejido, hay que identificar las partes, arrastrando hacia ellas el rótulo con su nombre. El programa indica si se hizo correctamente.

\section{b. Comentarios a favor}

- $\quad$ Es el laboratorio que cuenta con un contenido más claro y mejor explicado.

- Tiene los mejores dibujos.

- Las preguntas de evaluación están más relacionadas con el contenido tratado en el texto.

- Cuenta con actividades semejantes a la práctica real.

- El contenido es más variado, más ilustrativo y con mayor animación.

c. Comentarios en contra (minoritarios)

- Las imágenes observadas a través del microscopio no están muy claras.

- $\quad$ Posee poca información teórica.

- Presenta poco dinamismo.

- La interacción (arrastrar e identificar) es poca.

\subsection{Nutrición}

\section{a. Formato}

Se empleó una mascota virtual, Tami, para poder ilustrar el efecto que provoca la carencia o exceso de los nutrientes que consumimos en nuestras dietas (Fig. 3). También se ilustra la formación de moléculas orgánicas y cómo se procede en el laboratorio para su identificación (Fig. 4).

\section{b. Comentarios a favor}

- Presenta estructuras químicas que ayudan a comprender los contenidos 


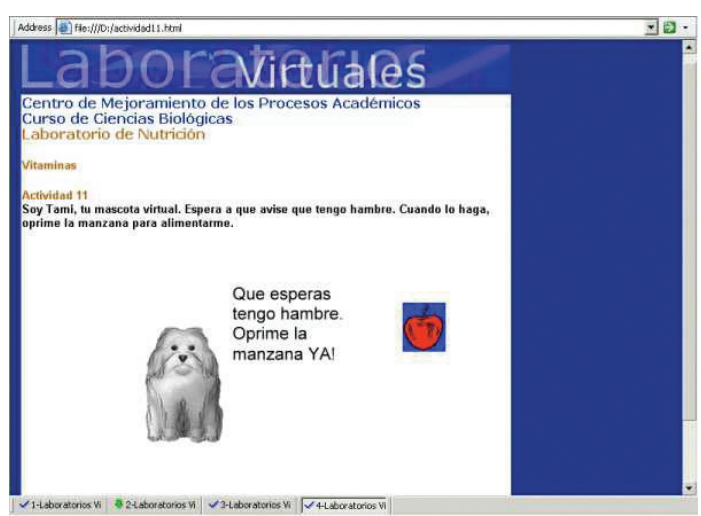

Fig. 3. Si a la mascota virtual, Tami, no se le alimentaba adecuadamente, sufría los síntomas de diversas enfermedades.

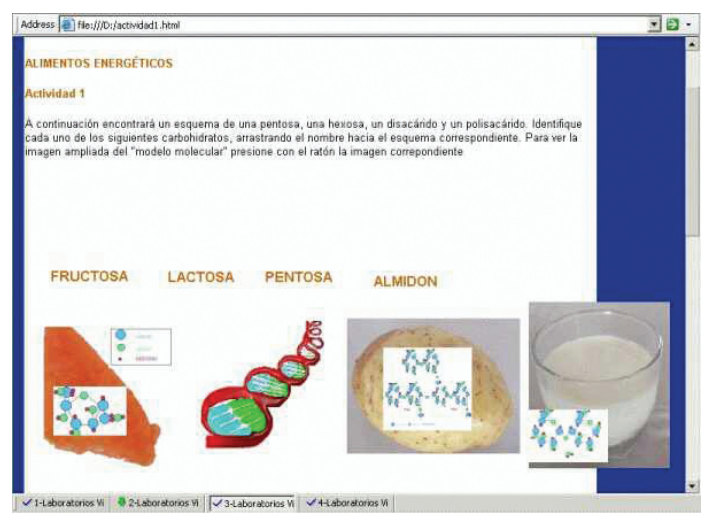

Fig. 4. Se facilitó el aprendizaje de la estructura química del almidón asociándola directamente con una imagen conocida: la de un alimento que lo contiene en grandes cantidades (la papa).

- $\quad$ Su elaboración denota buena creatividad.

- $\quad$ Es el más divertido, dinámico e ilustrativo.

\section{c. Comentarios en contra (minoritarios)}

- Las preguntas resultaron muy difíciles de responder.

- $\quad$ No se podía entender claramente lo que le pasaba a la mascota virtual cuando le faltaba un nutriente o se daba exceso de otros.
- El contenido no cuenta con la información suficiente.

- No se le encontró el valor didáctico a la mascota virtual.

\subsection{Digestión}

\section{a. Formato}

Se describen los tipos de alimento y los órganos que intervienen en los procesos de la digestión. La interacción incluye dar alimento a la persona para ver cómo se modifica éste al pasar por el sistema digestivo (Fig. 5).

Texto de la animación:

"En este esquema el bolo alimenticio es verde y los tractos digestivo y respiratorio son rosados. Note que el bolo alimenticio bajó por el conducto de la izquierda que esta conformado por la faringe (solo la parte inferior u orofaringe) y el esófago. El conducto de la derecha está conformado por la laringe y la tráquea, que se comunican con los bronquios, los bronquiolos, los alvéolos y los pulmones. Observe también que la epiglotis se mueve impidiendo el paso del bolo alimenticio hacia la laringe.

Fin de la actividad 3. Presione aquí para regresar a la página principal del laboratorio de digestión."

Con un vídeo endoscópico se ilustra la estructura gástrica.

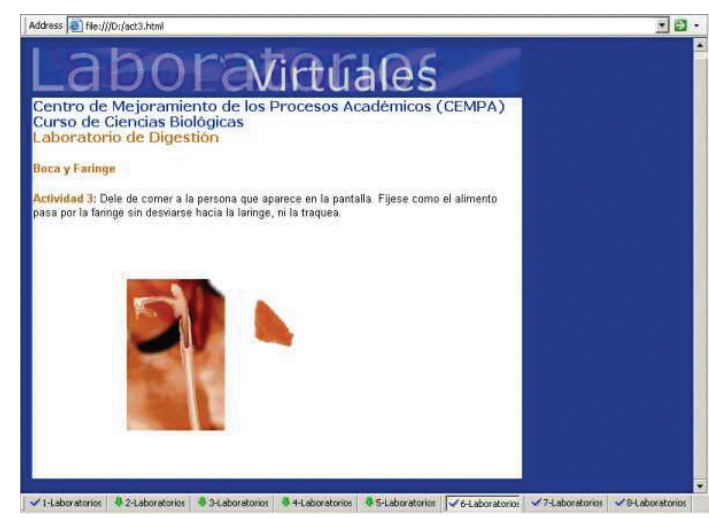

Fig. 5. Junto a esta imagen interactiva aparece el texto que explica cómo iniciar la animación. 


\section{b. Comentarios a favor}

- $\quad$ El contenido está desarrollado de una forma muy clara y precisa

- $\quad$ Está más relacionado con la vida cotidiana.

- Es más funcional para ser desarrollado.

\section{c. Comentarios en contra (minoritarios)}

- Faltan animaciones y dibujos más dinámicos.

\subsection{El mundo de las mariposas}

\section{a. Formato}

Presenta las generalidades de la biología de los artrópodos, con énfasis en los insectos. Se toma como base el comportamiento, reproducción y nutrición de las mariposas. Por ejemplo, la primera actividad (Fig. 6) dice "Observe cuidadosamente los siguientes especímenes (araña, caracol, piojo, pulga, mariposa, camarón, libélula, chapulín, planaria, medusa, estrella de mar). Arrastre hacia el centro del recuadro los que usted considere que son artrópodos. Hágalo de uno en uno. Cada vez que introduzca una figura al recuadro que no sea un artrópodo, sáquela" (la computadora indica cuando se ha cometido un error). También incluye alimentar orugas con diversos alimentos para ver si los aceptan y buscar mariposas ocultas en un bosque virtual interactivo.

\section{b. Comentarios a favor}

Usa una tecnología muy novedosa, al permitir buscar insectos ocultos en el panorama de 360 grados del bosque.

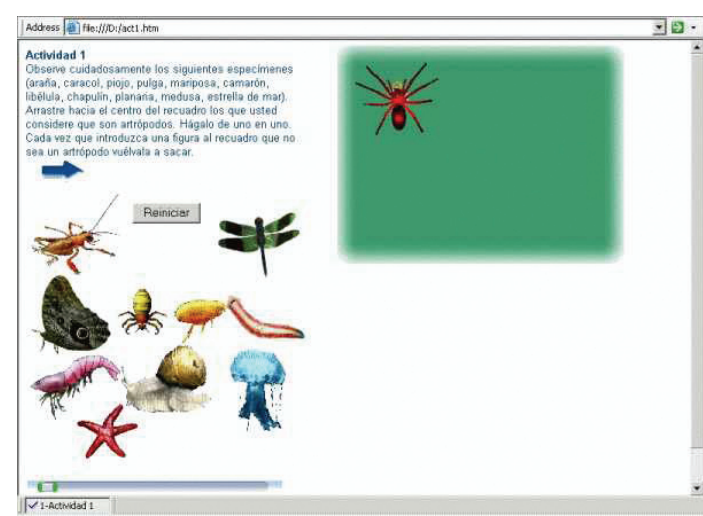

Fig. 6. Imágenes de invertebrados, que incluyen artrópodos y no artrópodos, y que deben seleccionarse interactivamente con base en las características aprendidas en la introducción teórica del laboratorio virtual.

\section{c. Comentarios en contra (minoritarios)}

La versión inicial traía preguntas cuya respuesta no tenía relación con la práctica del laboratorio y debía buscarse en libros especializados que no estaban al alcance del estudiantado de la UNED.

\section{Discusión}

\section{Ventajas del estudio}

Una ventaja clave del presente estudio es que cubre un periodo largo (seis años) y la totalidad de la población, apartándose de la práctica común de simplemente estimar las características de la población a partir de muestras. Esto aumenta nuestra confianza en que los resultados son indicadores de lo que se puede esperar en el uso de laboratorios virtuales entre la población estudiantil propia de la UNED. Todo esto es apoyado por estudios adicionales previos en la misma institución (Román 1996, Monge Nájera, 1998).

La distribución geográfica amplia de la población estudiada (todas las regiones de 
Costa Rica), la predominancia de mujeres y la edad media idéntica a la media característica del estudiantado de la UNED, da generalidad a los resultados y conclusiones a nivel de la educación a distancia en Costa Rica. Nuestros resultados coinciden con los obtenidos en otras latitudes. Por ejemplo, Marín y otros (2003) también tuvieron éxito en sustituir laboratorios reales que implicaban problemas de acceso, con laboratorios virtuales no muy diferentes en tecnología de los usados por nosotros, aunque en un contexto diferente (Europa). Por otra parte, aunque además del contexto el material era diferente, pues trabajaban con prácticas de ingeniería, Torres y otros (2003) tuvieron en general los mismos resultados en cuanto a ventajas y desventajas de los laboratorios virtuales. Aunque nosotros no distribuimos nuestros laboratorios mediante Internet, debido a que la política institucional es hacerlo en formato de disco compacto contra el pago de matrícula, los diseñamos de manera que bastaría copiarlos a un computador central ("servidor") para que puedan no solo ser descargados, sino operados completamente en línea. En este sentido, nuestros materiales calzan con todos los requerimientos indicador por McKee (2003) para ese propósito.

\section{Cumplimiento de objetivos}

El uso predominante que hace el estudiantado de la UNED de los laboratorios en computadoras ubicadas en la casa o en "cibercafés", indica que se cumplió el objetivo de proveer laboratorios virtuales que funcionaran incluso en equipos sencillos como los que predominan fuera de las instalaciones académicas. Esto, creemos, se debe a que tuvimos el cuidado de conocer previamente lo que estaban haciendo otros investigadores, como Schmid (1998) y Torres y otros (2001a, b), tanto en lo que se refiere a requisitos de compatibilidad como a la previsión del uso de los laboratorios mediante protocolos de Internet.
La calificación predominantemente buena sobre las instrucciones y el funcionamiento de los laboratorios también indica que se lograron dos objetivos importantes del proyecto de laboratorios virtuales: usar una plataforma tecnológica compatible con cualquier equipo sin necesidad de conexión a Internet y ser una opción competitiva en relación con los laboratorios reales (Monge Nájera, 2001).

Estos laboratorios virtuales fueron bien recibidos por la gran mayoría de quienes los usaron. No se requiere de mucha complejidad de programación (al momento de elaborar los laboratorios) para obtener una opinión favorable por parte de las usuarias y los usuarios, y los detalles mejorables afectan más a los contenidos y no tanto al medio digital en que se presentaron. En este sentido, experiencias como la descrita por Torres y otros (2004) para laboratorios virtuales en el área de física e ingeniería, calzan perfectamente con lo que ya habíamos concluido nosotros un lustro antes, trabajando con un campo diferente: la biología (Monge Nájera y otros, 1999).

\section{Requisitos mínimos que debe tener un laboratorio virtual: la opinión de las estudiantes y los estudiantes}

Una de las conclusiones más importantes que podemos obtener es la lista mínima de requisitos que debe tener un laboratorio virtual para ser exitoso, según un elemento fundamental: el propio estudiantado.

Las personas que usaron los laboratorios virtuales para aprender solicitan las siguientes características en un laboratorio virtual:

1. Variedad en los contenidos y actividades

2. Sencillez en la redacción y el uso

3. Creatividad en texto, prácticas y multimedios 
4. Dinamismo en la redacción y la presentación

5. Realismo en imágenes y actividades

6. Alta calidad en todo tipo de contenido gráfico (dibujos, fotografías, vídeos)

7. Preguntas realmente relacionadas con la práctica de laboratorio

8. Cierto grado de "diversión" en cuanto a su uso

9. Fuerte interactividad entre usuario(a) y laboratorio

Esta lista viene a completar de manera significativa lo que ya habían afirmado Stallings (2000) para el uso de computadoras para intercambio de información, y Torres y otros (2001) para las simulaciones especializadas en el área de la física.

Evaluación dentro del marco de referencia de los estándares internacionales.

Kappelman (2001) definió los siguientes requisitos como el estándar internacional para un buen laboratorio virtual:

\section{Autocontenido \\ 2. Interactivo \\ 3. Combinar imágenes bidimensionales y tridimensionales \\ 4. Tener animación, vídeo y sonido \\ 5. Incluir ejercicios \\ 6. Instalación automática \\ 7. Navegación no necesariamente lineal \\ 8. Posibilidad de guardar anotaciones digitales \\ 9. Buscador}

En este campo, los laboratorios de la UNED cumplen con los requisitos 1 a 7, faltando únicamente los de anotaciones digitales y buscador. Sin embargo, no consideramos que estos requisitos se justifiquen, por lo siguiente:

Los laboratorios virtuales se usan en combinación con otros recursos de computación, como procesador de texto (generalmente Word), que puede ser usado para guardar notas adjuntas al laboratorio. No nos parece que sea importante que las notas queden dentro del laboratorio como afirma Kappelman, en lugar de en un documento adjunto.

Adicionalmente, los sistemas operativos modernos tienen su propio buscador, por lo que no es necesario agregar otro.

\section{Confiabilidad de las respuestas al cuestionario: comparación con otra fuente de información}

Es importante validar o "triangular" los resultados de los cuestionarios con otras fuentes de información. Si hay coincidencia, aumenta la confianza en la validez de las respuestas que se recibieron al aplicar los cuestionarios. Aunque no formó parte de los objetivos del presente estudio, esa validación existe, pues nuestros resultados sobre la opinión de los estudiantes calzan con las opiniones expresadas por el personal docente, y publicadas en un informe anterior, según los cuales tanto la presentación gráfica, como la navegación, actividades interactivas y contenidos son considerados satisfactorios por al menos el $75 \%$ de las personas encuestadas (Monge Nájera y otros, 2003).

\section{Perfil de estudiantes que usaron los laboratorios}

Los estudiantes del período estudiado se matricularon en todos los centros de la UNED y la proporción de mujeres (55\%) fue ligeramente superior a la de hombres (45\%). De las mujeres el $42 \%$ solteras y de los hombres el $85 \%$. La mayoría de estudiantes se encuentran entre los 19 y 21 años y tienen acceso a computadoras e Internet, y saben usar procesador de textos y navegador de Internet. Estos aspectos se analizaron en detalle previamente (bibliografía en Monge Nájera y otros, 2001), por lo que no se detallan aquí. 


\section{Potencial para la socialización del aprendizaje}

Un aspecto central de los laboratorios virtuales, es que al estar basados en la misma tecnología que Internet, y particularmente por usar formato HTML (o XML) y un navegador web, se prestan muy bien para una socialización del aprendizaje. Para estudiantes conectados a Internet, sería posible intercambiar en tiempo real sus experiencias con los laboratorios. Este aspecto teórico no se ha llevado a la práctica porque constituye la parte esencial de un desarrollo futuro del programa de laboratorios virtuales de la UNED.

\section{Conclusión general}

El desarrollo y uso de cualquier laboratorio virtual con las características de los elaborados por la UNED y examinados en este estudio retrospectivo, tienen las siguientes ventajas:

- $\quad$ Es fácil y agradable ejecutarlos y las ilustraciones y demás aspectos gráficos son buenos.

- $\quad$ Ayudan a comprender los contenidos teóricos, pues permiten observar de cerca procesos que son difíciles de estudiar en la naturaleza.

- Las preguntas de evaluación sirven de refuerzo y motivación.

Las principales críticas, que fueron el no ser autocontenidos, la poca familiaridad con computadoras y el mucho tiempo que requieren los laboratorios virtuales, nos parecen de poca importancia, porque:

- Los laboratorios fueron probados y afinados con base en retroalimentación de quienes los usaron, por lo que tras la primera prueba se corrigió el defecto de no ser autocontenidos.

- En los años que han transcurrido desde estas primeras pruebas, el estudiantado de la UNED ha cambiado y actualmente la mayoría usa con frecuencia equipo de cómputo (UNED, datos internos sin publicar).

- $\quad$ Aunque no hemos estudiado formalmente el tiempo que se tarda en ejecutar los laboratorios virtuales, seguramente es inferior al de los reales si se toma en cuenta el tiempo que se tarda viajando para ejecutar los reales, y el hecho es que el estudiantado ha logrado hacerlos y se muestra mayoritariamente satisfecho con ellos.

Una crítica importante, que recibimos de una tutora, es que con los laboratorios virtuales no se adquieren las habilidades que proporciona el manejo de aparatos reales.

Nuestra respuesta es que, en su vida diaria, la mayoría de estas/os estudiantes no requerirán operar formalmente equipo de laboratorio, por tratarse en general de docentes de ciencias para primaria y secundaria.

La evaluación más importante que se puede hacer a estos laboratorios, es si con ellos se aprende. Al comparar los grupos que hicieron laboratorios reales con los que usaron laboratorios virtuales, no hubo diferencia estadísticamente significativa en la tasa de estudiantes que aprobaron el curso, a pesar de que ambos grupos hicieron los mismos exámenes (Méndez y otros, 2001). Los laboratorios virtuales resultaron por tanto comparables con la opción presencial, que es mucho más costosa para la UNED.

Los laboratorios virtuales elaborados por la UNED entre 1997 y 2004 han superado ya la etapa piloto y han demostrado ser una buena opción para quienes no pueden o no desean realizar prácticas en un laboratorio real (Monge Nájera, 1998). Son además una opción creativa, moderna y económica para ésta y otras instituciones universitarias, tanto a distancia como presenciales, que requieran laboratorios dentro de sus procesos de formación. 


\section{Otras conclusiones específicas}

- $\quad \mathrm{Al}$ analizarse un periodo largo (seis años) y la totalidad de la población, se tiene confianza en que el uso de laboratorios virtuales permite aprender y superar exitosamente las evaluaciones, en la población estudiantil propia de la UNED.

- La calificación del estudiantado a los laboratorios virtuales es predominantemente favorable, lo cual representa un gran apoyo para el desarrollo y aplicación de nuevos laboratorios virtuales en el futuro.

- $\quad$ No se requiere de mucha complejidad de programación al momento de elaborar los laboratorios, para obtener una opinión favorable por parte de las usuarias y los usuarios.

- Los detalles mejorables en los laboratorios, según la opinión del estudiantado, afectan más a los contenidos que al medio digital en que se presentaron. Esto se refiere fundamentalmente a los primeros laboratorios, pues esas fallas se corrigieron durante el proceso de retroalimentación y afinamiento.

- Es fundamental que los laboratorios virtuales tengan variedad en los contenidos y actividades; sencillez en la redacción y el uso, y creatividad en texto, prácticas y multimedios.

- Los requisitos mínimos de un laboratorio virtual incluyen dinamismo en la redacción y la presentación; realismo en las imágenes y actividades, y una alta calidad en todo tipo de contenido gráfico (dibujos, fotografías y vídeos).

- Las preguntas de los laboratorios virtuales deben estar realmente relacionadas con la práctica de laboratorio, permitir cierto grado de "diversión" en cuanto a su uso, y basarse en una fuerte interactividad entre usuario(a) y laboratorio.

\section{Agradecimientos}

Agradecemos a las estudiantes y los estudiantes, tutores y encargados de cátedra que participaron en el estudio, a Marta Rivas Rossi por toda su ayuda durante estos años de trabajo con los laboratorios virtuales, así como a Lizette Brenes Bonilla, Miguel González Castañón y un(a) revisor(a) anónimo(a) por valiosas sugerencias que permitieron mejorar un manuscrito anterior.

\section{Referencias bibliográficas}

Alejandro A., C. A. (2004). Prácticas de laboratorio de Física General en Internet. [Versión electrónica]. Revista Electrónica de Enseñanza de las Ciencias, 2(3), 1-5.

Arregui, M., Marín, R., y Sanz, P. J. (2003). Recent Educational Experiences by Using the UJI Online Robot. International Conference on Network Universities and e-Learning, (publicación digitial, sin paginación). Valencia, España: (s.e).

Bailey, M. y Lluetkehans, A. (1998, (s.f)). Guidelines for facilitating virtual learning teams in web-based instruction. Ponencia presentada en el $I X$ Congreso Internacional: Tecnología y Educación a Distancia. Cooperación Interamericana en Educación a Distancia, San José, Costa Rica: EUNED.

Bonvin, D., Gillet, D., Longchamp, R. y Salzmann, Ch., (1997). Telepresence: An Opportunity to Develop Practical Experimentation in Automatic Control Education. En Proceedings of the European Control Conference, (sin paginación). Brussels, Belgium: (s.e). 
Candelas F. A., Gil, P., Ortiz, F., Pomares, J., Puente, S. T. y Torres, F. A. (2003). Visual: Herramienta práctica para la enseñanza de Visión Artificial. $1^{a}$ Jornada en Educación y Práctica en Visión Artificial, (pp. 115-121). Mallorca: (s.e).

Candelas, F. A., Gil, P., Ortiz, F. G., Pomares, J. A. Puente, S. T. y Torres, F. A. (2003). Virtual Laboratory for Teaching Robotics. International Journal of Engineering Education, Special Issue "Remote Access / Distance Learning Laboratories”, 19, 363-370.

Candelas, F. A., Gil, P., Ortiz, F., Pomares, J. A., Puente, S. y Torres, F. A. (2004). El laboratorio virtual como herramienta en el proceso enseñanza-aprendizaje. Investigar Colaborativamente en Docencia Universitaria. En: Actas de las II Jornadas de Redes de Investigación en Docencia de la Universidad de Alicante, (sin paginación). Alicante: Editorial Club Universitario. http://aer.ual.es/publica es/Posters/PosterROBOTMOVIL.pdf

Candelas, F. A., Gil, P., Ortiz, F., Pomares, J., Puente, S. y Torres, F. A. (2003). Laboratorios Virtuales para el aprendizaje práctico de asignaturas de ingeniería. En: I Jornadas de Redes de Investigación en Docencia Universitaria, (sin paginación). España: Editorial Club Universitario.

Candelas, F. A., Navarrete, J. A., Pomares, J. A., Puente, S. T., Segarra, V., Torres, F. A. (2004, abril). A flexible JAVA Class Library for Simulating and Teleoperating Robots, 11 th IFAC Symposium on Information Control Problems in Manufacturing (INCOM04), Salvador de Bahía, Brazil.
Candelas, F. A., Ortiz, F. G. Pomares, J., Puente, S. T. y Torres, F. A. (2001a). Robolab: Laboratorio virtual de robótica básica a través de Internet, En: Segundas Jornadas de Trabajo sobre Enseñanza Via Internet/Web de la Ingeniería de Sistemas y Automática (EIWISA'01, (sin paginación). Madrid: http://www. disclab.ua.es/robolab/

Candelas, F. A., Ortiz, F. G., Puente, S. T., y Torres, F. A. (2002). Remote Robot Execution through WWW Simulation. 15th Int. Conference on Pattern Recognition (ICPR 2000), 4 , (pp. 503-506), España, Prentice Hall. http://csdl2.computer.org/persagen/ DLAbsToc.jsp?resourcePath=/dl/ proceedings $/ \&$ toc $=\mathrm{comp} /$ proceedings/icpr/2000/ 0750/04/0750toc.xml\&DOI=10.1109/ ICPR.2000.902967

Canovas, J., Mangas, J., Martínez Larraz, C., Puente, S. T. y Torres, F. A. (2001b). Web teleoperation of robots with simulation feedback. Telemanipulator and Telepresence Technologies VIII, Proceedings of SPIE, 4570, 105-112.

Cisneros, Ma. del P. (1998). El aprendizaje colaborativo con el empleo de Learning Space, caminado hacia una nueva cultura del trabajo en un ambiente virtual. IX Congreso Internacional: Tecnología y Educación a Distancia. Cooperación Interamericana en Educación a Distancia, (pp. 125-132). San José, Costa Rica: EUNED.

Cruz, A. (1998). La experiencia de los académicos en la aplicación de una herramienta de trabajo colaborativo: el caso de Learning Space en la UNED de Costa Rica. X Congreso Internacional sobre Tecnología y Educación a Distancia, (pp. 161-169). San José, Costa Rica: EUNED. 
Deering, M., Rushforth, K. y Sowizral, H. (2000). The Java 3D API Specification. ( $2^{\mathrm{a}}$ ed.). New York: Addison-Wesley.

Dormido, S., Sánchez, J. y Morilla, F. (2000). Laboratorios virtuales y remotos para la práctica a distancia de la Automática. XXI Jornadas de Automática, Conferencia plenaria, (sin paginación). Sevilla, España: s.e.

García, D., Sánchez F. M. y Sebastián, J. M. (2003). Remote-access education based on image acquisition and processing through the Internet, IEEE Transaction on Education, 46, 142 148.

Gillet, D., Latchman, H. A., Salzmann, Ch. y Crisalle, O. D. (2001). Hands-On Laboratory Experiments in Flexible and Distance Learning, Journal of Engineering Education, 90, 187-191.

Gillet, D., Mondada, F., Salzmann, Ch., y Saucy, P. (1999). Sharing of Unique or Expensive Equipment for Research and Education, Informatik / Informatique, Magazine of the Swiss Informatics Societies, 4, 32-33.

Kappelman, J. (2001).Virtual laboratories for physical anthropology. Nueva York, United States: Wadsworth Pub Co.

Laaser, W. (1998). Seminarios virtuales: ¿Cómo aplicarlos en educación a distancia? IX Congreso Internacional: Tecnología y Educación a Distancia. Cooperación Interamericana en Educación aDistancia (pp. LIII-LXII). San José, Costa Rica: EUNED.

McKee, G. T. (2003). An online robot system for projects in robot intelligence, International Journal of Engineering Education - Especial Issue Remote Access/DistanceLearningLaboratories, 19, 356-362.
Méndez Estrada, V. H. (1999a). Anatomía humana: estudio de los tejidos mediante el laboratorio virtual. $X$ Congreso Internacional sobre Tecnología $y$ Educación a Distancia, (publicación digital, sin paginación). San José, Costa Rica: EUNED.

Méndez Estrada, V. H. (1999b). Estudio a distancia de la reproducción sexual $\mathrm{y}$ asexual con un laboratorio virtual. X Congreso Internacional sobre Tecnología y Educación a Distancia, (publicación digital, sin paginación). San José, Costa Rica: EUNED.

Méndez Estrada, V. H., Monge Nájera, J. y Montero, M. (1999). El uso del correo electrónico en la UNED: la visión de quienes participaron en procesos de capacitación. Innovaciones Educativas, 11, 27-36.

Méndez Estrada, V. H., Monge Nájera, J. y Rivas Rossi, M. (2001). Laboratorios virtuales: qué son, por qué usarlos y cómo producirlos. San José, Costa Rica: EUNED.

Méndez Estrada, V. H., Monge Nájera, J. y Rivas Rossi, M. (2002). La evolución de los laboratorios virtuales durante una experiencia de cuatro años con estudiantes a distancia. XI Congreso Internacional sobre Tecnología y Educación a Distancia, (pp. 5-8). San José, Costa Rica: EUNED.

Méndez Estrada, V. H., Monge Nájera, J., Rivas Rossi, M. (1999). Internet, multimedia and virtual laboratories in a "Third World" environment: how we solved the 21 basic problems in the Costa Rican Distance Education University. X Congreso Internacional Sobre Tecnología y Educación a Distancia, (publicación digital, sin paginación). San José, Costa Rica: EUNED. 
Méndez, V. H., Monge Nájera, J. y Rivas Rossi, M. (2001). Internet, multimedia and virtual laboratories in a "Third world" environment. Open Learning, 3(16), 279-290.

Méndez, V. H., Monge Nájera, J. y Rivas Rossi, M. (2003). Enseñanza a distancia de estrategia empresarial: opinión de estudiantes y docente sobre el uso de material multimedia y recomendaciones para mejorar su eficacia. X Encuentro Iberoamericano de Educación a Distancia: Calidad, Tecnología y Valores en la Educación Superior a Distancia (pp. 21-23), San José, Costa Rica: EUNED.

Monge Nájera, J. (1998). La estrategia CIAC 2000. San José, Costa Rica: EUNED.

Monge Nájera, J. (1999). Cómo creamos un curso híbrido entre el WEB de Internet y el libro de texto tradicional para un curso libre sobre biodiversidad. X Congreso Internacional sobre Tecnología y Educación a Distancia, (publicación digital, sin paginación). San José, Costa Rica: EUNED.

Pensa, D. y Sabulsky, G. (1998). Sobre el uso de los media en los procesos de enseñanza. IX Congreso Internacional: Tecnología y Educación a Distancia. Cooperación Interamericana en Educación a Distancia, (pp. 484-497). San José, Costa Rica: EUNED.

Rivas, Marta. (1999a). La enseñanza de la digestión por medio de un laboratorio virtual. X Congreso Internacional sobre Tecnología y Educación a Distancia, 2, (publicación digital, sin paginación). San José, Costa Rica: EUNED.

Rivas, Marta. (1999b). La enseñanza de la nutrición por medio de un laboratorio virtual. $X$ Congreso Internacional sobre Tecnología y Educación a Distancia, 2, (publicación digital, sin paginación). San José, Costa Rica: EUNED.

Rodino, A. Ma. (1996/1997). Las nuevas tecnologías informáticas en la educación: viejos y nuevos desafíos para la reflexión pedagógica. VII Congreso Internacional sobre Tecnología $y$ Educación a Distancia, (pp. 51-71). San José, Costa Rica: EUNED.

Román, M. A. (1996). Mediciones pedagógicas y nuevas tecnologías de la comunicación de la información. VII Congreso Internacional sobre Tecnología y Educación a Distancia, (publicación digital, sin paginación). San José, Costa Rica: EUNED.

Schmid, Ch. (1998). Using the World Wide Web for Control Engineering Education, Journal of Electrical Engineering, 49, 205-214.

Stallings, W. (2000). Capítulo 19. En: Stallings, W., Comunicaciones $y$ Redes de Computadores ( $6^{\mathrm{a}}$ ed., p. 810), España: Prentice-Hall.

Suárez, J. C. (1998). Docencia tradicional y facilitación del aprendizaje: ruptura, continuidad o nuevo paradigma? En: IX Congreso Internacional sobre Tecnología y Educación a Distancia. Tomo I. Cooperación Interamericana en Educación a Distancia, 1, (publicación digital, sin paginación). Costa Rica: EUNED.

Tapani, J. (1998). Innovative anarcism online-investigating made-in-Japan virtual learning environments. En: $I X$ Congreso Internacional: Tecnología y Educación a Distancia. Cooperación Interamericana en Educación a Distancia, (pp. 346-356). San José: Costa Rica, EUNED. 